\title{
Design and Numerical Simulation using Vibration Analysis for the Detection and Reduction of Failures of the Gear Pair of the Differential System of a Powertrain
}

\author{
Cristhoper E. Jaimes Martínez, J. Flores Méndez*,(D, Gustavo M. Minquiz, \\ Pablo Gutiérrez Cruz, Fernando Medina Pérez, A. C. Piñón Reyes, \\ Janette Castro Hernández and René Pérez Pérez
}

In this work, a numerical methodology is implemented applying the computational finite element method for a pair pinion-crown of bevel gears in spiral of the differential system of a compact Sport Utility Vehicle (SUV), with the objective of establishing a criterion of the results to characterize the failure of the gear pair during its operation. To do this, from a CAD model obtained by 3D scanning, the numerical results of the structural case are compared by correlating the transient, fatigue, modal and harmonic studies between a pair of gears without damage and another pair with a damaged (chipped) tooth on the pinion. It is observed that from the harmonic response of stress and vibration, a criterion can be established to differentiate the new pair of gears from the damaged pair, the latter presenting a frequency response pattern with high values with respect to the first. The above may be a reference option for detecting the failure of spiral bevel gear pairs used in automobile differential system.

\section{Introduction}

Gears are the main components of the transmission systems of mechanical power. The spiral bevel gears are critical components of machines used in the vast majority of automotive differential gearboxes. The fatigue strength of each gear is significant due to the high demand of maximum power, safety and weight. The failure of a gear during the operation is reflected not only in a higher replacement or repair cost, but also in the system downtime. Therefore, the early detection of the equipment failure plays an important role in saving such costs. Due to advances in technology, many procedures have been developed to study the behavior of geared systems before the failure. The analysis by the computational finite element method is one of them, since it is widely used to carry out structural, fatigue, modal and harmonic analyzes of gear transmission systems. For example, in the eighties a software based on the finite element method was developed to calculate the stresses in the teeth of bevel and hypoid gears [1].

Tecnológico Nacional de México/l.T. Puebla, Av. Tecnológico No. 420, Maravillas, C.P. 72220, Puebla, Pue, México.

*Corresponding author:

E-mail: javier.flores@puebla.tecnm.mx; Tel: (+52 1) 2223160085

DOI: 10.5185/aml.2021.15699
Similarly, another approach developed an algorithm using a combination of finite elements and a surface integral method to predict the distribution of contact stresses and deformation in gear teeth, the results obtained were compared with other methods [2]. Also, fatigue by contact on the teeth surface and fatigue by bending at the root of the teeth have been analyzed [3], for this a threedimensional finite element model of a pair of spur bevel gears based on the cumulative fatigue criterion and the stress-life equation is proposed. Finding, that the maximum fatigue by contact occurs near of the pitch line and the maximum bending stress occurs at the highest point of contact of a single geared tooth. Demonstrating that the previous stresses are directly proportional to the operating torque. Recently, analysis and test results of a differential gearbox of an automobile have been reported [4-6]. The authors perform a static and dynamic analysis of various bodies of the Mahindra-Bolero car's differential gearbox assembly, determining the limiting torque at which the gearbox fails. On the other hand, they also propose an experimental arrangement to determine the durability due to fatigue of spiral bevel gears using an approximation based on noise and vibration. Also, they carry out tests on a pair of spiral bevel gears to verify their suitability according to your work requirements, executing several duty cycles before failure. Finally, another of his results consisted in measuring the change in the noise 


\section{Advanced Materials Letters https://aml.iaamonline.org}

characteristics and vibrations produced by the pair of spiral bevel gears after a specific number of duty cycles, which can be used as a basis for the early detection of gear failure. From the previous works, it can be seen that the finite element method analysis for spiral bevel gears is recent and not yet fully developed, in contrast to the cases of analysis involving straight tooth bevel gears. This may be due to the complex shape and geometry of spiral bevel gears, which makes very difficult to carry out modeling and analysis by computational mechanics. Therefore, as a further contribution in the field, in this work a numerical methodology is implemented applying the finite element method for a pair pinion-crown of bevel gears in spiral of the differential system of a compact Sport Utility Vehicle (SUV), the main objective consists in establish a new criterion based on the numerical results to characterize the failure of the gear pair during its operation.

\section{Methodology}

To estimate the durability or useful life and the early detection of the failure of the spiral bevel gear pair during its operation, a methodology is proposed that consists of comparing the numerical results of the computational finite element analysis between a pair of gears without any type of damage or wear respect to another pair with a damaged (broken) tooth on the pinion. The computational mechanics analysis consists of the modeling of the transient, fatigue, modal and harmonic analysis of the pair pinion-crown of bevel gears in spiral of the differential system of a SUV (Honda CRV-2000), see Fig. 1. The CAD design of the gear pair was carried out by 3D scanning of the physical components corresponding to the differential shown in Fig. 2, the number of teeth on the pinion is 8 and on the crown is 39 .
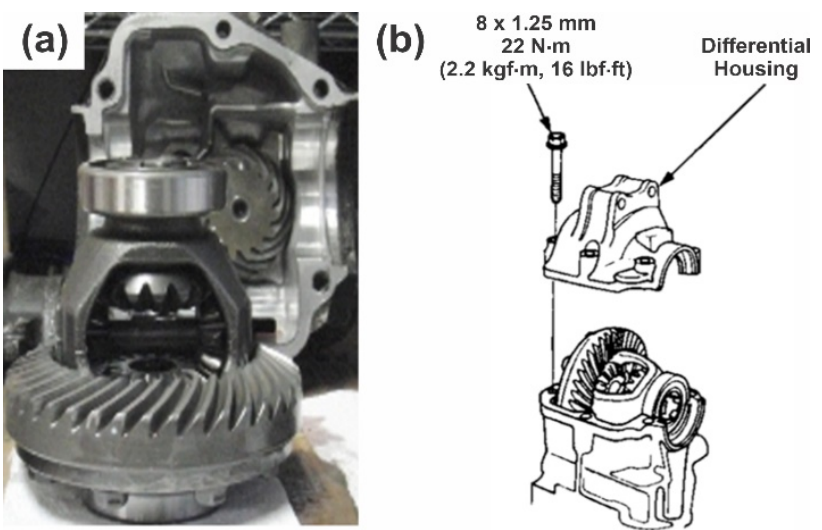

Fig. 1. (a) Real assembly of the differential system of a SUV and (b) view of the pair pinion-crown of bevel gears in spiral [7].

The computational finite element simulation was carried out considering primarily the following parameters: the contact surfaces between the teeth of the pinion and the crown were defined "without separation", for the axis of the pinion and the center of the crown they are considered frictionless supports and for fatigue analysis the load cycle is totally reversible applying Goodman's theory. For the transitory analysis, the input movement is applied to the pinion and in 5 seconds it will move $120^{\circ}$, for a torque applied to the pinion of $1000 \mathrm{~N} \cdot \mathrm{mm}$. The most used material for spiral bevel gears in car differential boxes is the steel AISI / SAE 4140. It is a high tensile strength steel with the following composition and mechanical properties [8]: Carbon- $0.4 \%$, Manganese- $0.8 \%$, Chromium- $0.95 \%$ and Molybdenum- $0.25 \%$; maximum tensile strength $1000 \mathrm{MPa}$, yield strength $800 \mathrm{MPa}$ and density $7.9 \times 10^{3} \mathrm{~kg} / \mathrm{m}^{3}$. The limit of the S-N curve for estimating cycles to failure due to fatigue is $N<10^{6}$ cycles [9].
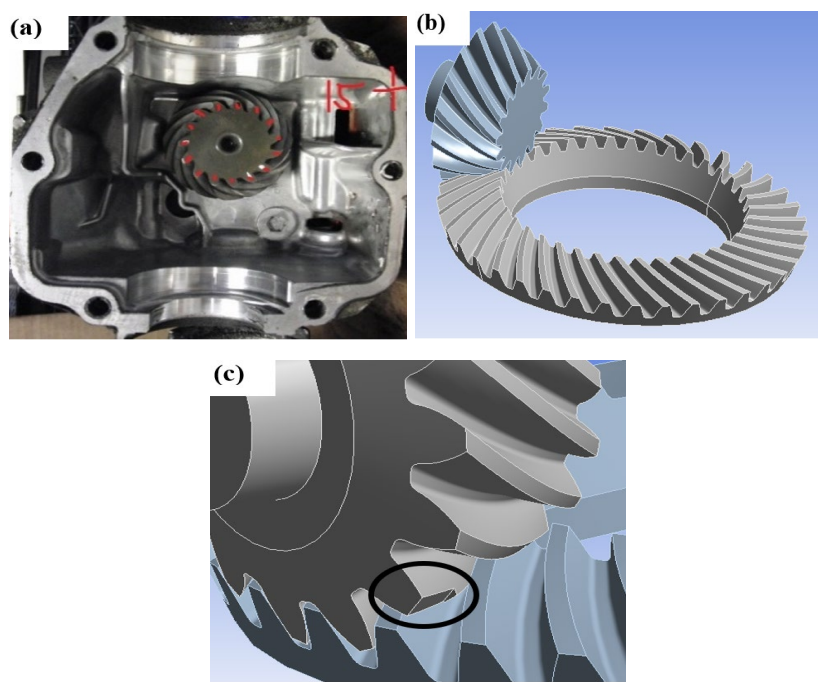

Fig. 2. (a) Real physical component corresponding to the pinion, (b) CAD model of the pair pinion-crown of bevel gears in spiral obtained by $3 \mathrm{D}$ scan and (c) model of the gear pair with a damaged (broken) tooth on the pinion.

\section{Discussion of results}

\section{Transitory analysis}

From this analysis, it is found that the maximum average von-Mises stress in the pair of spiral bevel gears without damage is $650 \mathrm{MPa}$ and occurs at the edge of the pinion teeth that is in contact with the crown (Fig. 3(a)). For the case of the damaged gear pair, the maximum average value of the von-Mises stress exceeds $800 \mathrm{MPa}$ (Fig. 3(b)). From this, it is clear that the part that is most prone to failure in a spiral bevel gear pair is the pinion. Note that the damaged gear pair has a higher stress magnitude.

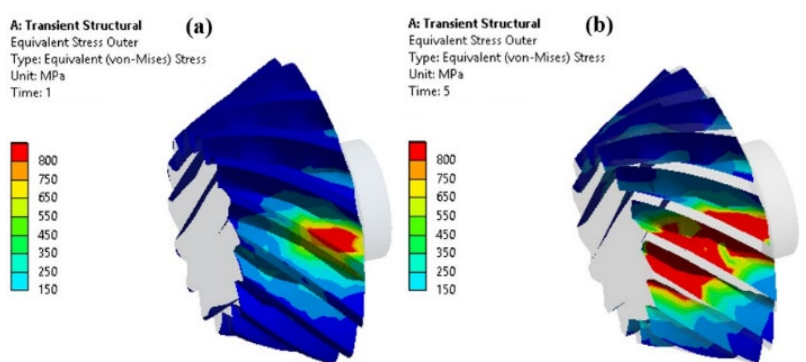




\section{Advanced Materials Letters https://aml.iaamonline.org}

Fig. 3. (a) Maximum von-Mises stress on the pinion of the undamaged gear pair and (b) Maximum von-Mises stress on the pinion of the damaged gear pair.

\section{Modal analysis}

The fundamental natural frequency for the undamaged gear pair was found to be $365.16 \mathrm{~Hz}$ (Fig. 4(a)), while for the pair with a broken pinion tooth it was $420.76 \mathrm{~Hz}$ (Fig. 4(b)). Comparing the natural frequency values, it can be seen how the frequency of the damaged gear pair is $55.6 \mathrm{~Hz}$ more than that of the undamaged gear pair, which shows that the characteristic vibration of the undamaged gear pair with respect to the damaged one will be different when are measured experimentally. Within the framework of this analysis criterion, the numerical simulations show the increase in the difference of frequency values, $2273.9 \mathrm{~Hz}$ and $3715 \mathrm{~Hz}$ for the second and third vibration modes, respectively (see data in Fig. 9). On the other hand, in Fig. 4 it is interesting to note for the case of the damaged gear pair the increase in the distribution of the critical deformation mapping. Therefore, by continuously comparing and measuring noise and vibration, the opportune detection of gear failure can be realized. Consequently, the physical condition of the pinion-crown pair (new, used or damaged) can also be known by comparing the noise and vibration characteristics.

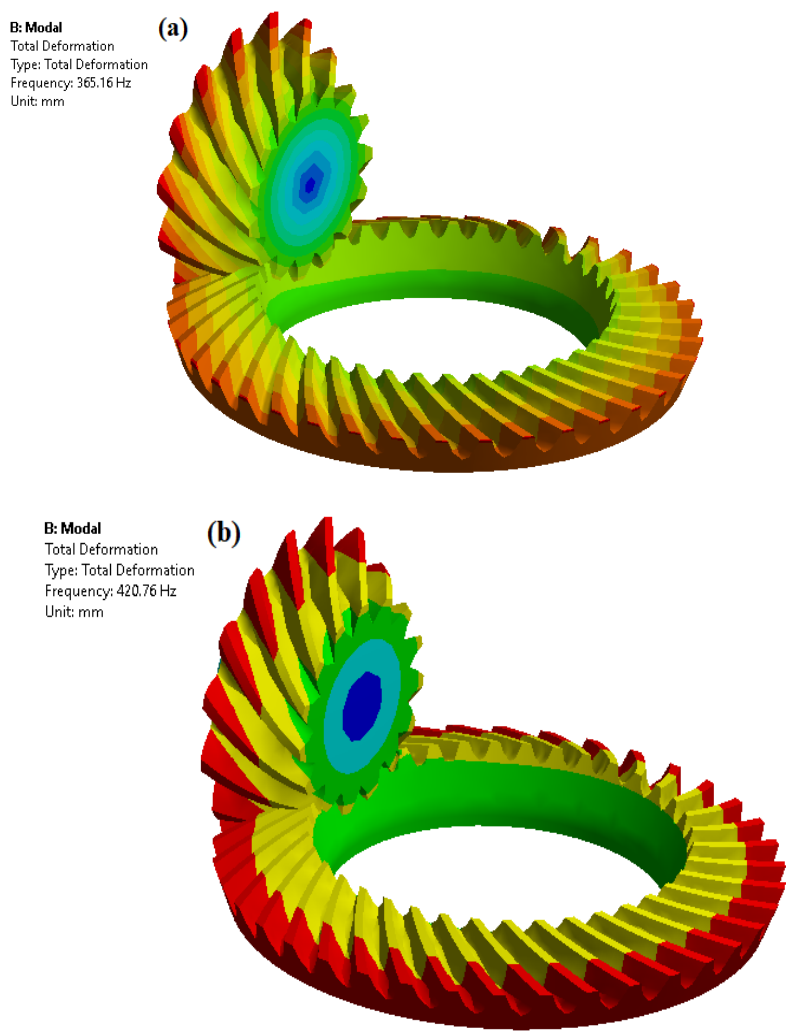

Fig. 4. (a) Fundamental natural frequency and critical deformation mapping of the pair of bevel gears without damage and (b) Fundamental natural frequency and critical deformation mapping of the pair with a damaged tooth on the pinion.

\section{Fatigue analysis}

The fatigue analysis is performed with a zero-based load cycle, because when the teeth gear, each tooth experiences a bending load and when disgear the load is released and becomes zero. From this analysis, for the pair of gears without damage, it is found that the number of cycles that the pinion can withstand before failure is $10^{6}$ cycles, which, for several authors, is considered as an infinite life, with an average safety factor of 3 (see Fig. 5). The value of the maximum equivalent alternating stress is $176 \mathrm{MPa}$ (see Fig. 6). Regarding the pair of gears with damage, Fig. 7 shows an average life of $10^{5}$ cycles (finite life) and an approximate maximum dominant safety factor of 1 . With an average value of alternating stress that exceeds $800 \mathrm{MPa}$ in the contact area between the teeth and at the base of the pinion shaft, shown in Fig. 8. Based on the previous results, it is important to note that, for the damaged pair of spiral bevel gears, its mechanical components may fail due to breakage prematurely under the action of fluctuating stresses, whose values may even be much lower than the yield point of the material, that is, the material may fail without its internal stress level having reached the critical values corresponding to those caused by stresses of the type static. This also makes it possible to differentiate the new gear pair from the damaged pair.

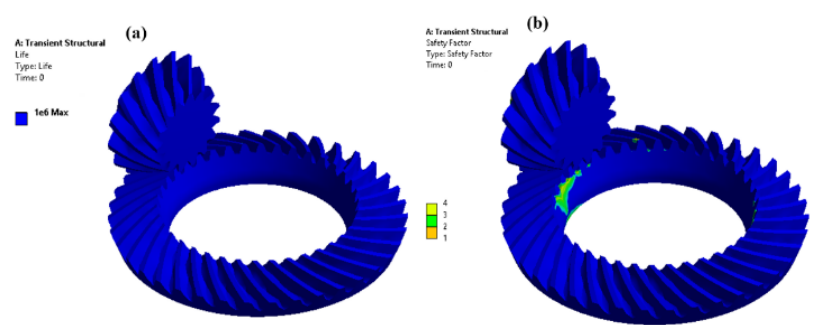

Fig. 5. (a) Number of cycles to the failure and (b) Fatigue safety factor of the pair of spiral pinion-crown bevel gears without damage.

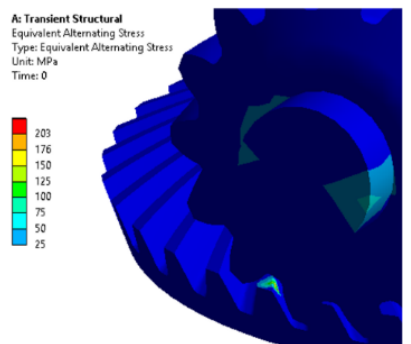

Fig. 6. Equivalent alternating stress of the pair of spiral pinion-crown bevel gears without damage.

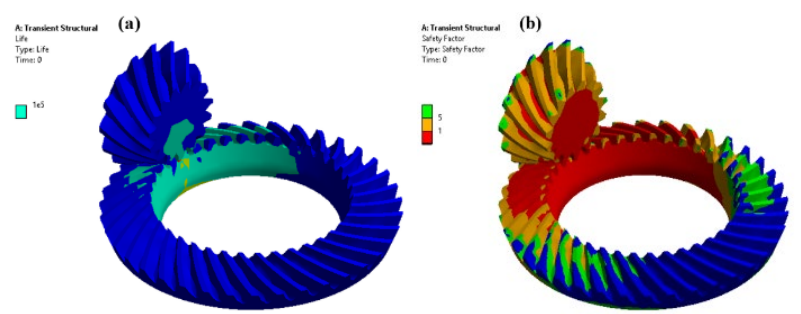

Fig. 7. (a) Number of cycles to the failure and (b) Fatigue safety factor of the pair of spiral pinion-crown bevel gears with a damaged tooth on the pinion. 


\section{Advanced Materials Letters \\ https://aml.iaamonline.org}

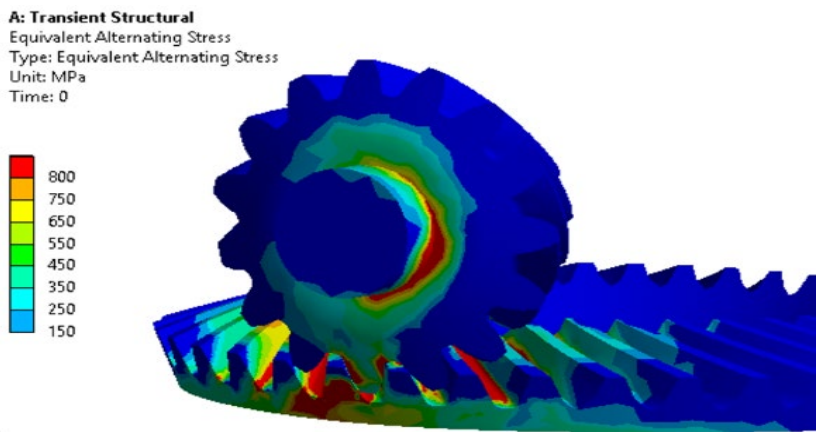

Fig. 8. Equivalent alternating stress of the pinion-crown spiral bevel gear pair with a damaged tooth on the pinion.

\section{Harmonic response}

The values of the resonance frequencies obtained from the modal analysis for the first 3 modes of vibration (sometimes, the only desired modes are those corresponding to the lower frequencies because they may be the predominant modes in the vibration of the system) of the pairs bevel gears without damage and with a damaged tooth on the pinion are shown in Fig. 9, respectively. And the comparison of the harmonic response between both pairs of gears is shown in Figs. 10 and 11. From the graphs, it can be seen that the amplitudes of the vibration $\left(1 \mathrm{G}=9.81 \mathrm{~m} / \mathrm{s}^{2}\right)$ and of the stress present a greater magnitude for the damaged gear pair and this is very pronounced in terms of the characteristic peak of the second mode of each gear pair. Notice that the acceleration and stress frequency amplitude response for the third mode of vibration increases (see case no-damage), this trend is due to the increase in frequency. For the case with-damage, numerical convergence could not be reached, but it is possible to observe this trend with the results shown in previous sections (see deformation mapping in modal analysis). Regarding the first mode, we can notice in both cases a very small peak value with respect to the second and third mode, it can be seen that the magnitude of the peak for the pair of new gears is approximately double with respect to the damaging pair. Then, the numerical simulations show that from the harmonic response, the new gear pair can be differentiated from the damaged one. This comparison fulfills the main objective of this research, which is to design and establish a simulation methodology using computational mechanics to estimate the useful life and early detection of failures for a pair pinion-crown of bevel gears in spiral of the differential system of an automotive powertrain during operation. From which it can be concluded that by comparing the characteristic values of noise and vibration between pairs of new and damaged spiral bevel gears, a criterion for the detection of their failure can be obtained. (a)

Tabular Data
\begin{tabular}{|l|l|l|}
\hline & Mode & $\sqrt{ }$ \\
\hline 1 & 1. & 365.16 \\
\hline 2 & 2. & 2201.2 \\
\hline 3 & 3. & 3018.8 \\
\hline
\end{tabular}

(b)

Tabular Data
\begin{tabular}{|l|l|l|}
\hline & Mode & $\sqrt{ }$ \\
\hline 1 & 1. & 420.76 \\
\hline 2 & 2. & 4475.1 \\
\hline 3 & 3. & 6733.8 \\
\hline
\end{tabular}

Fig. 9. (a) Resonance frequencies for the first 3 vibration modes of the pairs of spiral bevel gears without damage and (b) with damage.

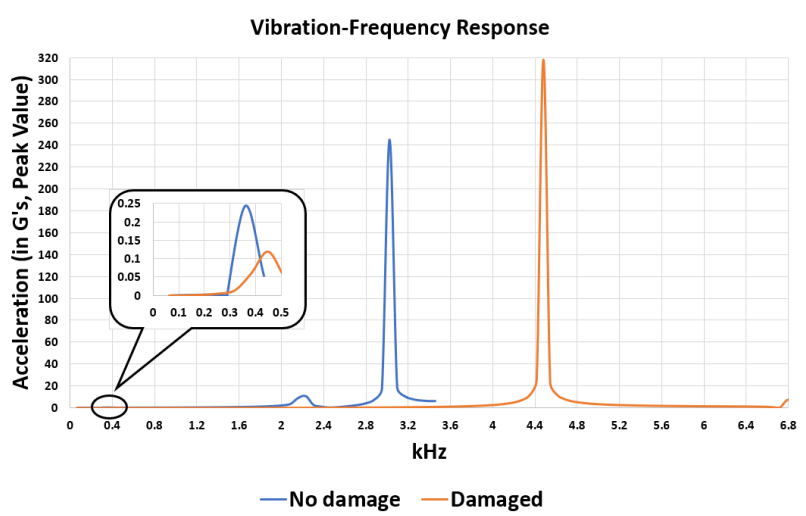

Fig. 10. Vibration-Frequency amplitude response.

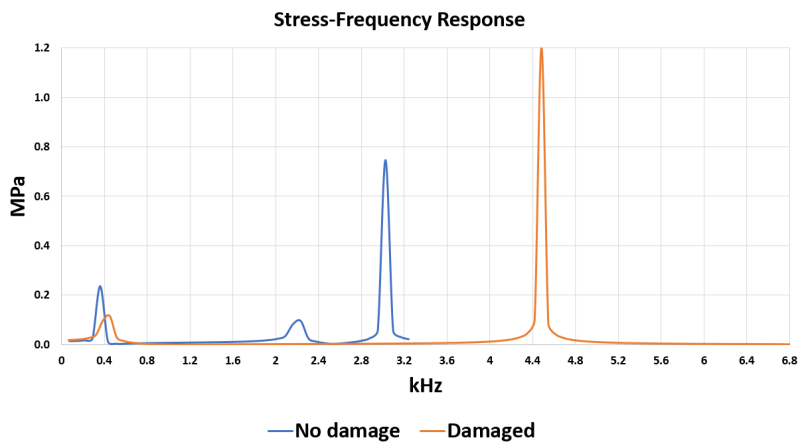

Fig. 11. Stress-Frequency amplitude response.

\section{Conclusion}

From the above results, for the case of a new pair of spiral bevel gears used in the differential system of a Sport Utility Vehicle (SUV), the equivalent maximum von-Mises stress was found to be $650 \mathrm{MPa}$, which is maintained within the permitted limits, since the yield strength of AISI 4140 steel is $800 \mathrm{MPa}$. From fatigue analysis, the gear pair showed infinite life, a safety factor of 5 , and a maximum equivalent alternating stress of $176 \mathrm{MPa}$. In contrast to the results obtained for a pair of spiral bevel gears with a damaged tooth on the pinion, which indicate stress states outside the elastic limit of the AISI 4140 steel and failure due to premature rupture under the action of fluctuating stresses (finite life $10^{5}$ cycles and approximate safety factor of 1 ). From the modal analysis, the fundamentals natural frequencies of the new gear pair with respect to the damaged pair differed by $55.6 \mathrm{~Hz}, 2273.9 \mathrm{~Hz}$ and $3715 \mathrm{~Hz}$ for the first 3 vibration modes, respectively. The above demonstrated that by comparing the characteristic values of noise and vibration it is possible to detect a system failure. Namely, the stress and vibration frequency response diagrams of the harmonic analysis showed that the maximum (peak) amplitude values are higher for damaged gears than for new ones. Therefore, the method proposed in the present work allows stablish the foundations for the detection of failures of pairs of spiral bevel gears used in the gearbox of the differential system of an automobile through the use of computational mechanics. All of the 


\section{Advanced Materials Letters https://aml.iaamonline.org}

above even allows to generate a record of spectra as the transmission system suffers wear and observe its evolution in more detail, this fact leads to the reduction of failure by allowing to implement preventive maintenance measures during the operation of the mechanical system.

\section{Acknowledgements}

The authors acknowledge the funded support from "Tecnológico Nacional de México/Instituto Tecnológico de Puebla", for providing us the use of the Scanning equipment and licensed Finite Element Analysis software for all modeling works.

Conflicts of interest

All the authors declare that there is no conflict of interest.

\section{Keywords}

Spiral bevel gears, Finite Element Analysis (FEA), transitory analysis, fatigue analysis, modal analysis, harmonic analysis, durability.

Received: 19 May 2021

Revised: 13 July 2021

Accepted: 15 July 2021

\section{References}

1. Wilcox, Lowell; Gear Technology, 1985, 1, 9.

2. Vijayakar, S.M.; Houser, Donald R.; Gear Technology, 1993, 4, 26.

3. Song, Deng; Lin, Hua; Xing-hui, Han; Song, Huang; Journal of Central South University, 2013, 20, 279.

4. Bhavi, Iresh; Kuppast, Vinay; Kurbet, Shivakant; Int. Journal of Engineering Research and Application, 2017, 7, 49.

5. Bhavi, Iresh; Kurbet, Shivakant. Multi Body Dynamic analysis of Differential gear box of an Automobile, Proc. 2011, ANSYS India Users Conference, 2011

6. Bhavi, Iresh; Kuppast, Vinay; Kurbet, Shivakant; Applied Mechanics and Materials, 2016, 852, 545.

7. Honda Motor CO., LTD. (Ed. 1); Service Manual CR-V 1997-2000; U.S.A, 1997.

8. Fletcher Building CO., LTD. (Ed. 1); Easy Steel - Special Steel Book; New Zealand, 2020.

9. Boyer, Howard E. (Ed. 1); Atlas of Fatigue Curves; ASM International, U.S.A, 1986.

Authors biography

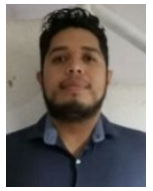

Cristhoper E. Jaimes Martínez, is student of Mechanical Engineering at the "Instituto Tecnológico de Puebla", México. With interest in the areas of material mechanics, manufacturing processes, machine element design and computational finite element analysis.

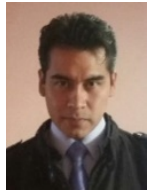

J. Flores Méndez, is Doctor in Materials Science. In addition, he has a Master in Mechanical Engineering. Currently, he is Professor-Researcher in the College of Metal-Mechanics Engineering at the "Instituto Tecnológico de Puebla", México. He is part of the National System of Researchers of Mexico. His research area focuses on studying the acoustic and electromagnetic response in metamaterials and computational finite element analysis.

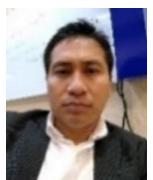

Gustavo M. Minquiz, received the Master's and Doctorate degree in Mechanical Engineering. Currently, he is Professor-Researcher in the College of Metal-Mechanics Engineering at the "Instituto Tecnológico de Puebla", México. His research area focuses on studying the energy saving and efficiency in manufacturing processes and computational finite element analysis.

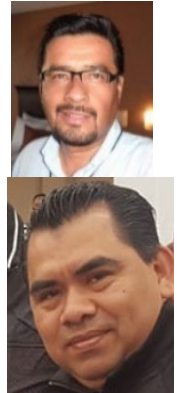

Pablo Gutiérrez Cruz, is Mechanical Engineer and Master in Engineering Sciences. He is Full-Time Professor-Researcher and Department head of the Industrial Engineering College of the "Instituto Tecnológico de Puebla", México. His field of research is the dynamics of machines and the analysis and synthesis of mechanisms.

Fernando Medina Pérez, holds the title of Industrial Engineer and the degree of Master in Engineering Sciences. He is FullTime Professor-Researcher in the College of Industria Engineering at the "Instituto Tecnológico de Puebla", México. His research area focuses on studying new mechanical and electromagnetic properties in materials for industrial applications.

A.C. Piñón Reyes, is Mechanical Engineer and Master in Mechanical Engineering. She is currently carrying out the research "Modeling and Simulation of Optical Properties for solar cells", to obtain the degree of Doctor in Sciences. She is Professor-Researcher in the College of Industrial Engineering at the "Instituto Tecnológico de Puebla", México. Her research area focuses on the study of new mechanical, electrical and electromagnetic properties of materials in optical devices for industrial applications.

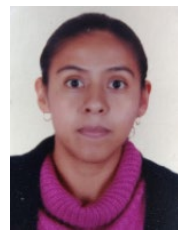

Janette Castro Hernández, is Industrial Engineer and Master in Integrated Manufacturing Systems and Quality Strategies. She is Professor-Researcher in the College of Industrial Engineering at the "Instituto Tecnológico de Puebla", México. Her research area focuses in improving and optimizing product designs, manufacturing processes and production systems through software systems.

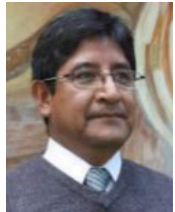

René Pérez Pérez, is Mechanical Engineer and Master in Mechanical Engineer. He is Full-Time Professor-Researcher and Department head of the Metal-Mechanics Engineering College of the "Instituto Tecnológico de Puebla", México. His research area focuses on studying new mechanical and electromagnetic properties in materials for industrial applications.

\section{Graphical abstract}

Starting from a CAD model of a pair pinion-crown of bevel gears in spiral of the differential system of a compact Sport Utility Vehicle (SUV) obtained by 3D scanning, a numerical methodology by analyzing harmonic vibration spectra is implemented applying the computational finite element method with the objective of establish a criterion of the results to characterize the failure of the gear pair during its operation.
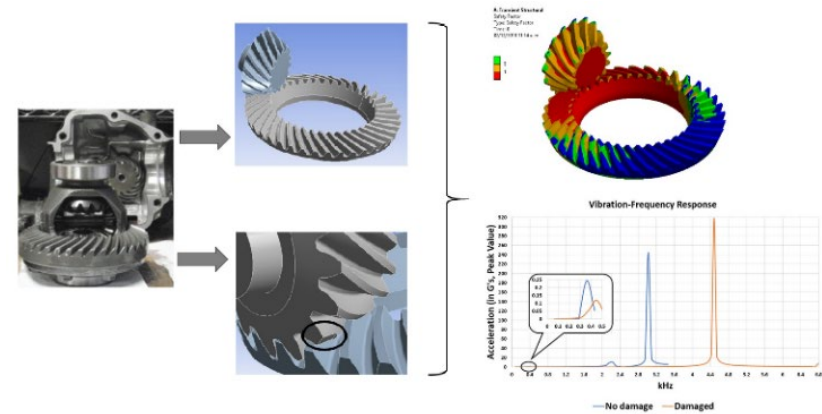